\title{
Improved Analytical Characterization of Solid Waste Forms (glass, metals, soils) by Fundamental Development of the Laser Ablation Technology
}

\author{
Richard E. Russo, Principal Investigator \\ Tel: 510/486-4258, Fax: 510/486-7303, email: RERusso@Ibl.gov \\ Lawrence Berkeley National Laboratory \\ Mail Stop 70 - 193A \\ Berkeley, CA 94720
}

\section{EXECUTIVE SUMMARY}

Laser ablation is a promising technology for chemical characterization within every DOE EM major problem area (high-level waste tanks, contaminant plumes, D\&D activities, spent nuclear fuel, mixed wastes, landfills, nuclear waste disposal, and HEU disposition). This EMSP research endeavors to expand the fundamental basis in laser ablation technology for its application to these DOE characterization needs. Laser ablation must be understood on a fundamental level to ensure confidence in chemical characterization of environmental samples. The goal is to develop a fundamental understanding of laser ablation processes, and to determine the influence of these processes on analytical behavior (sensitivity and accuracy) in order to bring this technology to fruition. This report summarizes the research completed in the first year of this project. The initial work addressed: accuracy of chemical characterization by verifying that the ICP (inductively coupled plasma) was not adversely influenced by ablated mass; accuracy of ablation sampling versus laser pulse time; and sensitivity enhancements through the use of various gas environments. The research and development utilized an existing ICP-AES system. Part of the effort also included the evaluation, purchase, and installation of an ICP-MS system. Three scientific manuscripts were completed and submitted to technical journals. One of the goals of this work is to support the efforts at all the National Laboratories investigating laser ablation technology for the management of DOE radioactive, hazardous chemical, and mixed waste; collaborations with scientists at other National Laboratories have been initiated.

\subsection{INTRODUCTION}

Laser ablation is a promising technology for chemical characterization within the EM program for numerous reasons. Laser ablation is a direct sampling and characterization technology; one ablates the sample and characterizes its chemical composition. The sample can be homogeneous, heterogeneous, radioactive, stable, inorganic, organic, biological, sludge, saltcake, soil, etc. Within the EM program, many of the samples will be hazardous. Laser ablation technology provides these capabilities. There is no sample preparation; therefore, laser ablation can eliminate thousands of dissolutions, eliminate additional solvent waste, minimize contamination of equipment, and minimize personnel exposure to samples and solvents. Chemical analysis using laser ablation requires a smaller amount of sample (< micrograms) than that required for conventional solution nebulization (milligrams). Depending on the analytical detection system, picogram to femtogram quantities may be sufficient for analysis, providing a pseudo " nondestructive" analytical technology. Laser ablation can be used in the laboratory or 
in a field environment; it represents one of the only potential methods to perform in-situ realtime characterization of metals in diverse complex samples. The goal of this EMSP project is to develop the fundamental and experimental capabilities so that laser ablation technology can be applied to EMSP chemical characterization needs. The three separate sections below describe completed research and development in FY97.

\subsection{EXPERIMENTÁL LASER ABLATION SYSTEM}

The experimental system for laser ablation and analysis is shown in Figure 1.The beam from a pulsed laser is directed into the "ablation" chamber and focused onto the sample surface. The ablated sample vapor is transported by gas flow to the central channel of an ICP torch. Spectral emission from the ICP is measured by a monochromator with a CCD detector. An inductively coupled plasma-mass spectrometer (ICP-MS) was purchased for this research. ICP-MS is utilized throughout the chemical analysis community because of its enhanced sensitivity and isotopic capabilities, two important parameters to the DOE chemical characterization mission. The initial research using this new instrument was obtained in the last month of the fiscal year; these data will be reported in the next annual report.

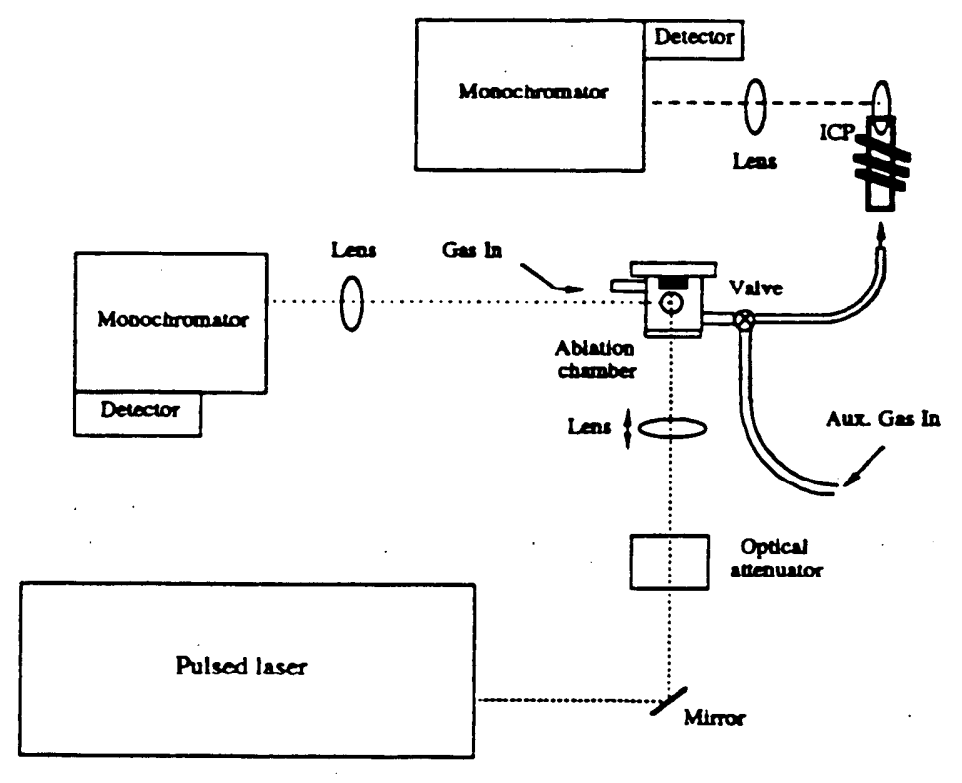

Figure 1. Diagram of LA-ICP-AES experimental system for chemical characterization.

\subsection{Verification of ICP-AES ACCURacy toAblated Mass}

Oneof the first studies we performed was to determine if the ablated sample entering the ICP affected the plasma conditions. It is critical to ensure that the ablated mass entering the ICP does 
not significantly influence plasma temperature and electron number density, because changes in these parameters will be manifested as changes in chemical concentration or composition; i.e., inaccurate analysis. For conventional ICP-AES analysis of solutions by means of liquid nebulization, the amount of aerosol entering the plasma is determined by the nebulizer/spray chamber parameters and does not significantly change during aspiration of standards and samples with slightly different concentrations. In contrast, the mass loading to the ICP during laser ablation sampling depends on the sample matrix and laser properties. Different amounts of ablated mass and/or dry aerosol of different particle sizes will be introduced to the ICP during laser ablation solid sampling of standards and samples.

The influence of ablated mass on the electron number density and temperature within the ICP was studied by measuring the ratio of $\mathrm{Mg}$ ionic to atomic emission lines and selected $\mathrm{Fe}$ lines, respectively. The ICP electron number density was investigated by using measurements of the $\mathrm{Mg}$ ionic to atomic resonant line ratios during laser ablation of an aluminum matrix. The ICP excitation temperature was measured by using selected Fe lines during laser ablation of an iron matrix. A Nd:YAG laser (3 ns pulse duration) at $266 \mathrm{~nm}$ with different repetition rates was used for these ablation sampling studies. Laser energy, power density, and repetition rate were varied in order to change the quantity of ablated mass into the ICP. The ICP emission intensity, which is assumed to be proportional to the amount of ablated mass, changed over approximately one order of magnitude during sampling of $\mathrm{Al}$ and Fe matrices. A priori, self absorption for the spectral lines used in the measurements was always checked.

The experimental system was described above. Elemental spectral emission was measured over the entire vertical length of the ICP. The ICP operated at gas flow rates of $14 \mathrm{l} / \mathrm{min}$ for Ar plasma gas, $1.0 \mathrm{l} / \mathrm{min}$ for auxilarygas and $0.2 \mathrm{l} / \mathrm{min}$ for central carrier gas. The Nd:YAG laser was operated at $266 \mathrm{~nm}$ with $3 \mathrm{~ns}$ pulse duration. Laser repetition rate was $10 \mathrm{~Hz}$ unless otherwise specified. The laser energy used in these studies was $5 \mathrm{ml}$ and $10 \mathrm{~mJ}$, andwas monitored continuously during the experiments. The reproducibility of the laser energy from shot to shot was approximately $5 \%$.

The samples consisted of either an aluminum matrix with a small amount of $\mathrm{Mg}(95 \% \mathrm{Al}, 4 \%$ $\mathrm{Cu}, 1 \% \mathrm{Mg}$ ) or pure Fe samples (99.999\%). These samples were chosen strictly to verify the ICP behavior; environmental samples are expected to behave similarly, but would present more complex spectra and significantly complicate these studies. The Al sample was chosen for the measurements of the ratio of magnesium ion to atom resonance lines. The pure Fe sample was used in measurement of the ICP temperature. A pure $\mathrm{MgO}$ (Pure Tech Inc., 99.9\%) sample was used to demonstrate self-absorption effects.

\subsubsection{Measurement of the Mg Ionic to Atomic Emission Ratios}

It is well known that the ionic to atomic spectral intensity ratio for the same element can be used to measure the "energy characteristics" of the ICP discharge. The fundamental expressions for calculating these parameters are beyond the scope of this report and can be found in Ciocan (under submission). The data in Figure 2 show this ratio over the entire vertical extent of the ICP for different laser power densities. The Mg II 279-nm/Mg I285changes by more than a factor of along the ICP central axis. This change corresponds to the axial inhomogeneities of the ICP temperature and electron number density. However, there are no significant changes of this ratio at different laser power densities at any height above the load coil. The scatter of the 
data in the region below the load coil are due to the weak $\mathrm{Mg}$ atomic emission line intensity in this region. The possibility of self absorption of these spectral lines was checked. This determination is essential, especially when working with resonant lines or even with nonresonant lines that belong to the matrix. The ratio remains constant over this range oflaserpower densities, proving that both resonant $\mathrm{Mg}$ lines are optically thin and no self absorption results. The Mg II 279-nm and Mg II 280-nm lines are almost " ideal" to checkfor self absorption, since they have very close upper excitation levels.

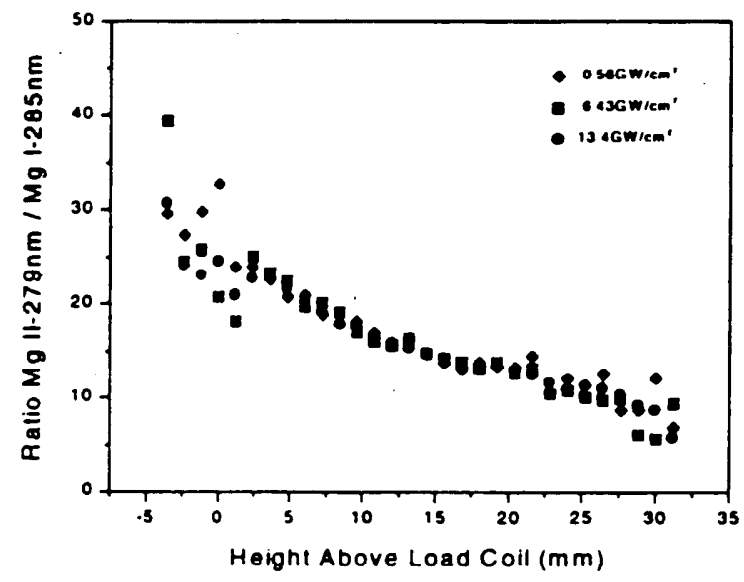

Figure 2. Ratio of Mg ionic to atomic emission in the ICP versus height above load coil for several laser power densities.

If the ICPis not perturbedbythe ablated mass, plasma temperature should not change. In this case, the optical emission signal will be proportionaltothe amount of ablated mass entering the ICP. In Figure 3 the ratio of $\mathrm{Mg} \mathrm{II} / \mathrm{Mg}$ I is plotted versus the relative intensity of $\mathrm{Al} \mathrm{I} 257-\mathrm{nm}$ which is proportional to the amount of ablated mass. The ratio remains constant within the error over approximately an order of magnitude change in the $\mathrm{Al}$ emission intensity. Al I 257-nm was selected as an indication of the total ablated mass, sinceitis a matrix line and the standard deviation from shot-to-shot ablation is less than for a minor component such as $\mathrm{Mg}$. In addition, the concentration of $\mathrm{Mg}$ is given over a large range (0.2\%-0.8\%)inhomogeneitiesin the can be expected to contribute to the error. The stronger $\mathrm{Al}$ emission line is a more reliable indication of the ablation process, as long as the line is not self absorbed. Here, the AIl 257-nm has been confirmed to be free of self-absorption. The line used for checking self absorption was Al 1 I265nm,whichhas a very closeupper state energy with Al I 257-nm. 


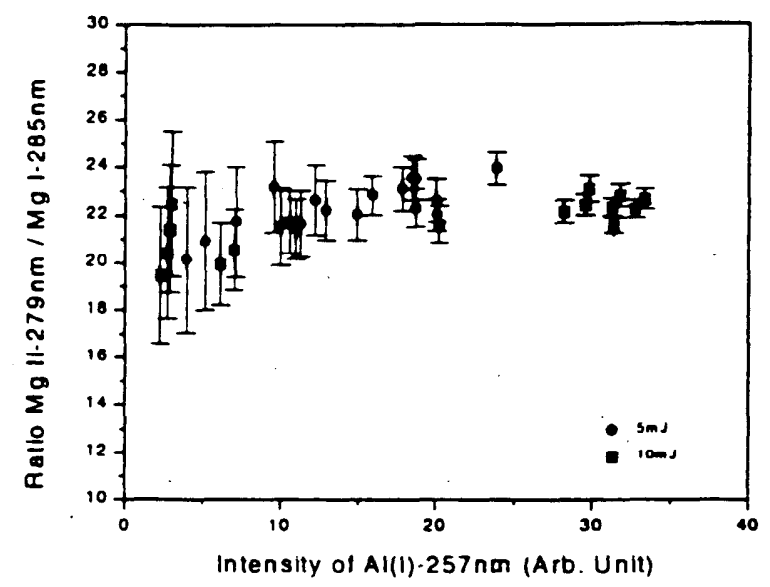

Figure 3. Ratio of Mg ionic to atomic emission versus the ICP Al emission line intensity.

In previous studies from this research group, roll-off and nonlinear dependence were observed in ICP intensity normalized to laser spot area, versus laser power density. If the roll-off and nonlinear dependence arc not caused by changes in ICY conditions due to mass loading, then theseICP data represent fundamental laser ablation processes. The ratio of $\mathrm{Mg} \mathrm{II} / \mathrm{MgI}$ versus laser power density was the same over the range of laser power densities used in this experiment. These data confirm that the roll-off and nonlinear dependenceare caused by laser ablation sampling and not by changes in the ICP conditions.

\subsubsection{ICP excitation temperature measurements}

The ICP temperaturecalculated during ablation using the different laser power densities is shown in Figure 4. Here weshowtheerror bars arising only from measurement uncertainties, rather than error bars reflecting uncertaintics in Einstein coefficients. ICP excitation temperature dots not change within the experimental errors. However. nonlinear mass ablation rate and rolloff were observed during laser ablation sampling of the Fe sample over this power density range the ablation rate changes by twoorders of magnitude ICP temperature remains constant over this power densityregion supportingourbelief that the roll-off and nonlinearity arc not caused by changes in ICP conditions from differentamounts of mass. These results are also supported by other nonspectralemission-basedmeasurementsin which roll-off and nonlinearity were observed.

In order to increase the amount of ablated mass entering the ICP, the laser repetition rate was varied from 5 to $50 \mathrm{~Hz}$. at a constant laser power density. The ratio of Fe I 386.0-nm to Fe I 381.6-nm exhibits a constant value within experimental

e $n$ t e r i n g t h e p la s m a i s i n c r e a s e d b y a factor of 10. The ICP temperature was measured under two experimental conditions for increasing the ablated mass: 1) fixed repetition rate of $10 \mathrm{~Hz}$ with increased power density, and 2 ) fixed power density with increased repetition rate. For both cases, the temperature was not influenced by mass loading. 


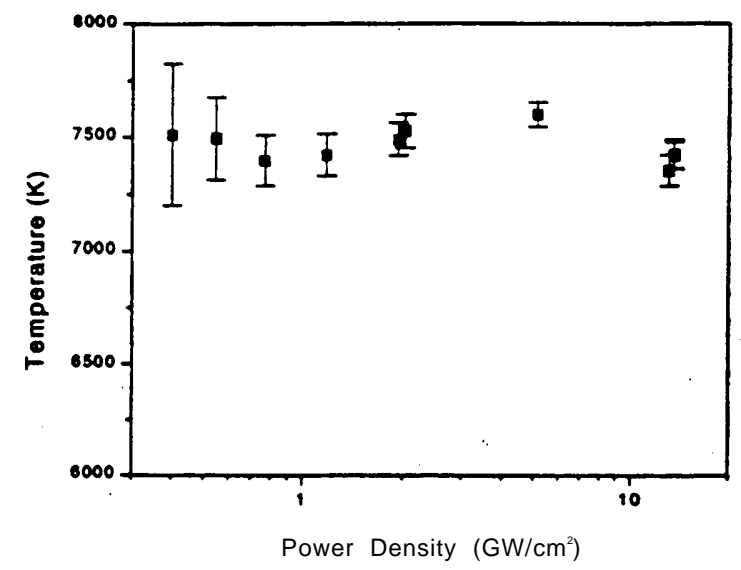

Figure 4. Excitation temperature in the ICP versus laser power density calculated using two line method.

\subsection{Time-Resolved Studies OF Mass Effects On ICP-AES}

The quantity of ablated mass and its relative composition of elemental components strongly depend on the number of laser pulses at the sample surface (Borisov, under submission). For chemical analysis, the quantity of mass removed determines the' signal intensity measured in the ICP-AES; a change in quantity can be' misinterpreted as a change in chemical concentration, The mass ablation rate (quantity) versus time is an important parameter and is influenced by the sample surface oxide layer, extended heating and melting of the sample, and crater formation. These parameters can be studied by measuring the ICP-AES emission intensity as a function of time during repetitive ablation sampling. Preferential vaporization (fractionation of components based on volatility) during laser ablation sampling can be a problem for chemical analysis. The thermal component of laser ablation sampling influences the relative composition of the vapor, enhancing low melting-point elements in the vapor phase; the vapor is not stoichiometric and the analysis is inaccurate. Experimental conditions under which the composition of the ablated vapor is representative of the bulk sample need to be elucidated for accurate chemical analysis. Quantity and relative composition versus continuous laser sampling were investigated in this part of the work. The mass ablation rate and ablated mass composition were studied by monitoring the time dependence of emission intensity in the ICP during repetitive laser ablation at a single location on the sample. The ratio of emission lines gives an indication of the relative composition of constituents.

For this part of the work, a brass sample was ablated using several lasers with various conditions. The lasers used are the KrF excimer with a 30-ns pulse width, Nd:YAG with a 3-ns pulse width, and Nd:YAG with a 35-ps pulse width. Laser ablation was accompanied by nebulization of water. Aqueous aerosol and ablated vapor were combined at an exit port of the ablation chamber using a T-connector and directed into the ICP for analysis. By nebulization of brass standard solution, the $\mathrm{Cu}$-to- $\mathrm{Zn}$ ratio was used as a reference for laser ablation experiments. $\mathrm{Cu}$ 224.7-nm and $\mathrm{Zn} 206.4-\mathrm{nm}$ ionic emission lines were used in all these experiments. 


\subsubsection{Time-resolved studies of mass ablation-rate dependence on laser fluence}

The data in Figure 5 show $\mathrm{Cu}$ II 224.7-nm line emission intensity time profiles. Generally, higher laser fluence provides higher overall ICP emission intensity, which corresponds to a larger mass ablation rate. $\mathrm{Cu}$ emission intensity stabilized after about 2 rnin when the brass sample was ablated with 308-nm excimer (30-ns) laser pulses (Fig. 5a). However, at the lowest fluence of $0.84 \mathrm{~J} / \mathrm{cm}^{2}, \mathrm{Cu}$ emission intensity increases slowly and stabilizes at values larger than those for higher fluences of 2.40 and $3.24 \mathrm{~J} / \mathrm{cm}^{2}$. This behavior may be explained by an increased degree of elemental fractionation at the low fluence. At a fluence of $0.84 \mathrm{~J} / \mathrm{cm}^{2}$, sampling (ablation) takes place from a large surface area. Melting and preferential vaporization of $\mathrm{Zn}$ can result in significant enrichment of $\mathrm{Cu}$ in the crater. $\mathrm{Cu}$ enrichment was confirmed by energy-dispersive X-ray spectroscopic (EDX) measurements performed in the laser ablated crater. After about 4 minutes of continuous ablation, sampling takes place from a $\mathrm{Cu}$-rich surface. Zn emission intensity shows the opposite behavior (not shown here), confirming this phenomena.

By using the 3-ns pulses from the Nd:YAG laser (Figure 5b), Cu emission intensity stabilizes after 20-30 s of ablation at low fluences, indicating a constant or steady rate of mass ablation. However, at higher fluences of 4.40 and $15.0 \mathrm{~J} / \mathrm{cm}^{2}$, emission intensity increases continuously as the crater is formed. $\mathrm{Zn}$ intensity (not shown here) follows that of $\mathrm{Cu}$, indicating an increase in the mass ablation rate with time. This behavior can be attributed to a "small spot effect". At these high fluences, the crater becomes narrow and deep (approximately $50 \mathrm{~m}$ in diameter and 1 $\mathrm{mm}$ in depth) after $3 \mathrm{~min}$ of ablation. It may be that molten droplets are ejected into the Ar flow due to the high pressures at the bottom of the crater. Alternatively, for deep craters the laserinduced plasma can form directly inside the crater and can be responsible for increased mass ablation rate, by sputtering or radiative heating from the plasma.

Ablation with 35-ps laser pulses resembles the 3-ns case, with indication of a "small spot effect" at higher fluences (Fig. 5c). At low fluences, time profiles of $\mathrm{Cu}$ emission intensity have a peaked shape, which is not characteristic of ablation with 3-ns laser pulses. The peak-shaped mass ablation rate behavior may result from the influence of the crater aspect ratio on the vapor phase expulsion of mass. In addition, as the crater develops, the fluence effectively changes and, hence, changes of the mass ablation rate can occur.

\subsubsection{Zn-to-Cu Mole Ratio Time Profiles}

Figure 6 compares time-resolved $\mathrm{Zn} / \mathrm{Cu}$ mole ratios measured during ablation with the different lasers at various fluences. Generally, after approximately 30-40 s of ablation, the ratios stabilize, indicating constant composition of the vapor phase. However, at low fluence of 0.84 $\mathrm{J} / \mathrm{cm}^{2}$ for 30-ns excimer laser ablation, the plume is initially enriched with $\mathrm{Zn}$ and the ratio only slowly 'decreases to its constant value as the sample surface becomes $\mathrm{Cu}$-enriched. A characteristic feature of ablation with the 30-ns pulse excimer laser is a large spread for $\mathrm{Zn} / \mathrm{Cu}$ ratios, especially at low fluences, indicating a strong thermal interaction (Figure 6a). The y-axis in Figure 6a is a log scale compared to the linear axis for Figures $6 \mathrm{~b}$ and $\mathrm{c}$. The ratios are much more stable and less dependent on fluence when ablation was performed with the 3-ns and 35-ps Nd:YAG lasers. Although the mass ablation rate at high fluences increases with time (Figs.5b 
and $\mathrm{c}$ ), the $\mathrm{Zn} / \mathrm{Cu}$ ratio is relatively stable, indicating small time variations of vapor phase composition. However, in all the cases, the ratio of elements in the vapor phase is highly influenced by the laser fluence.

A)

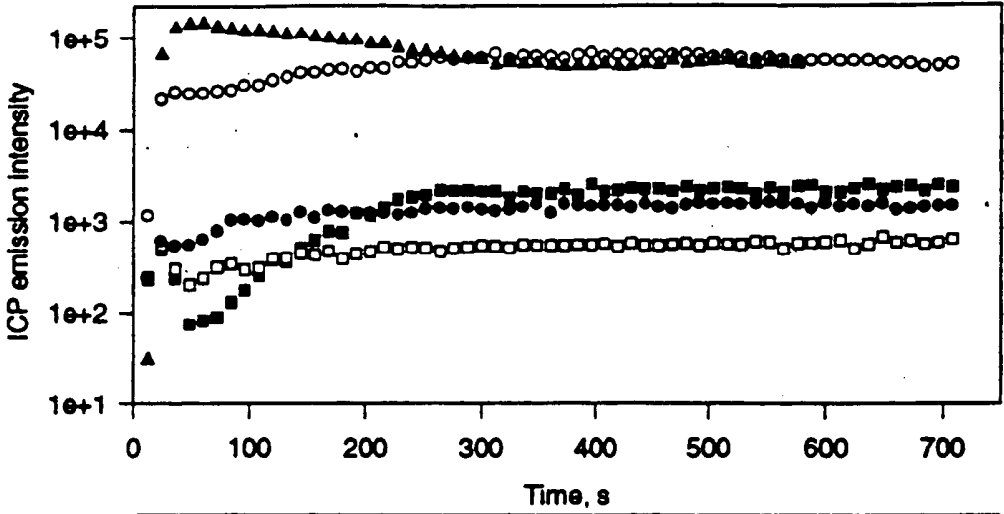

$$
\begin{aligned}
& -0.84 \mathrm{Jam}^{2} \\
& \text { - } 2.40 \mathrm{J \textrm {cm } ^ { 2 }} \\
& \text { - } 3.24 \mathrm{~J} \mathrm{~cm}^{2} \\
& \text { - } 5.40 \mathrm{~N} \mathrm{~cm}^{2} \\
& -24.0 \mathrm{~J} \mathrm{~cm}^{2}
\end{aligned}
$$

B)
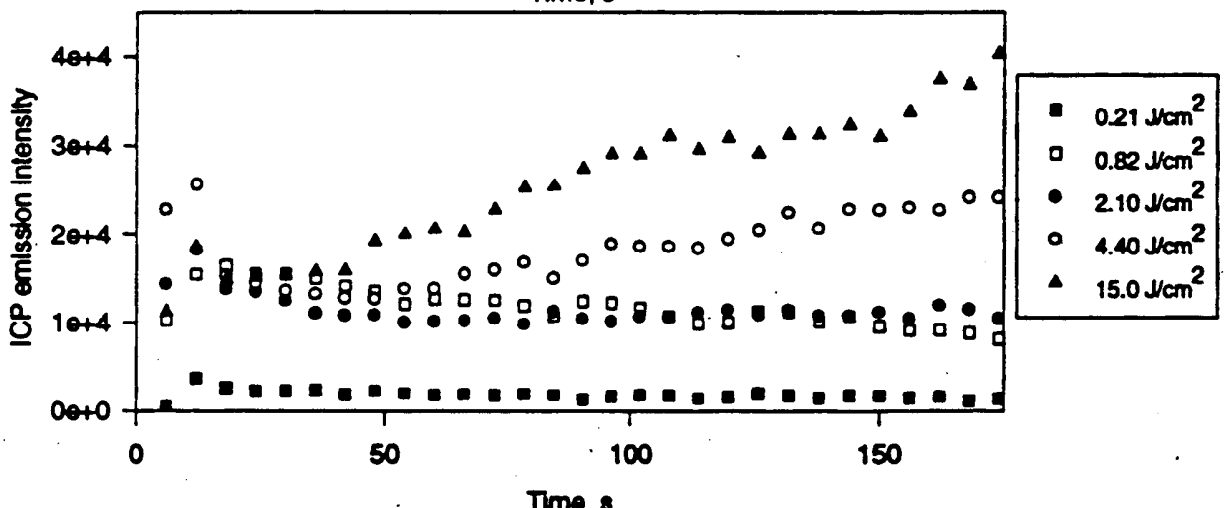

C)

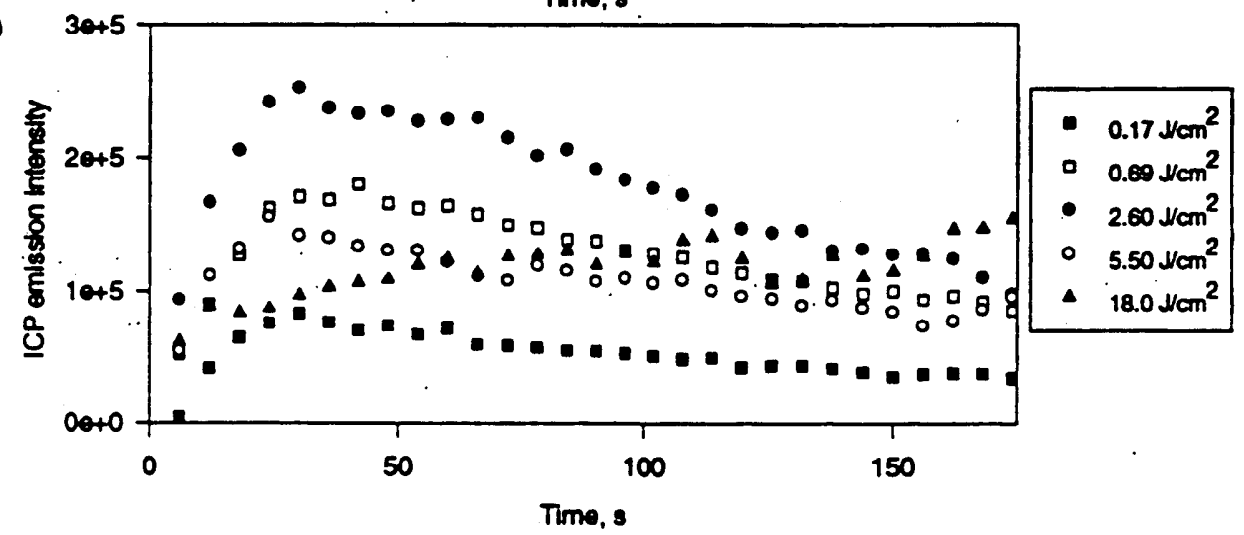

Figure 5. Cu (II) 224.7-nm ICP-AES emission intensity time profiles, measured during continuous ablation with 30-ns KrF excimer (A), 3-ns Nd:YAG (B), and 35-ps Nd:YAG (C) lasers at various fluences as indicated in the legends. Each point on the graph is an integrated for $6 \mathrm{sec}$ ICP intensity. 

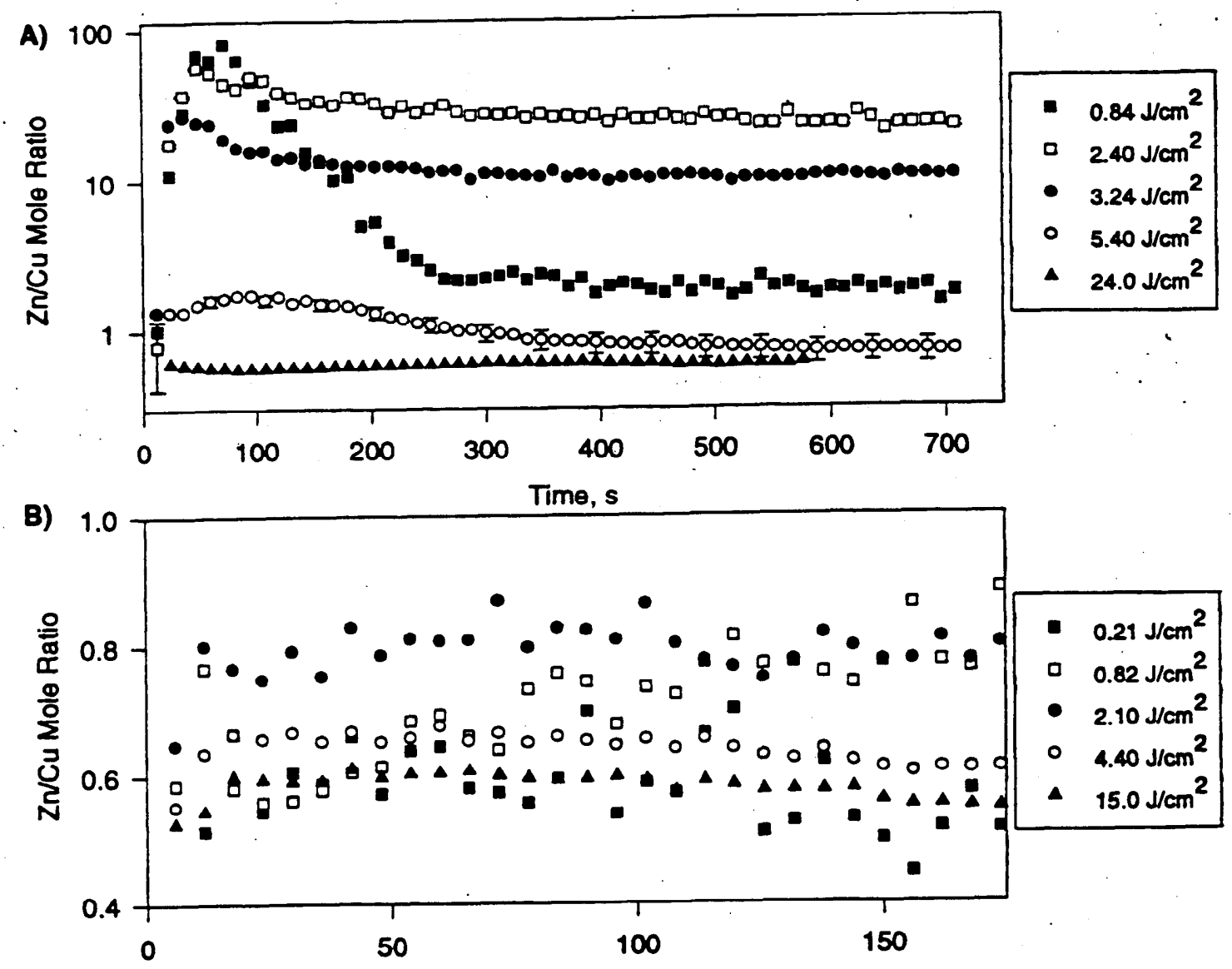

Time, s

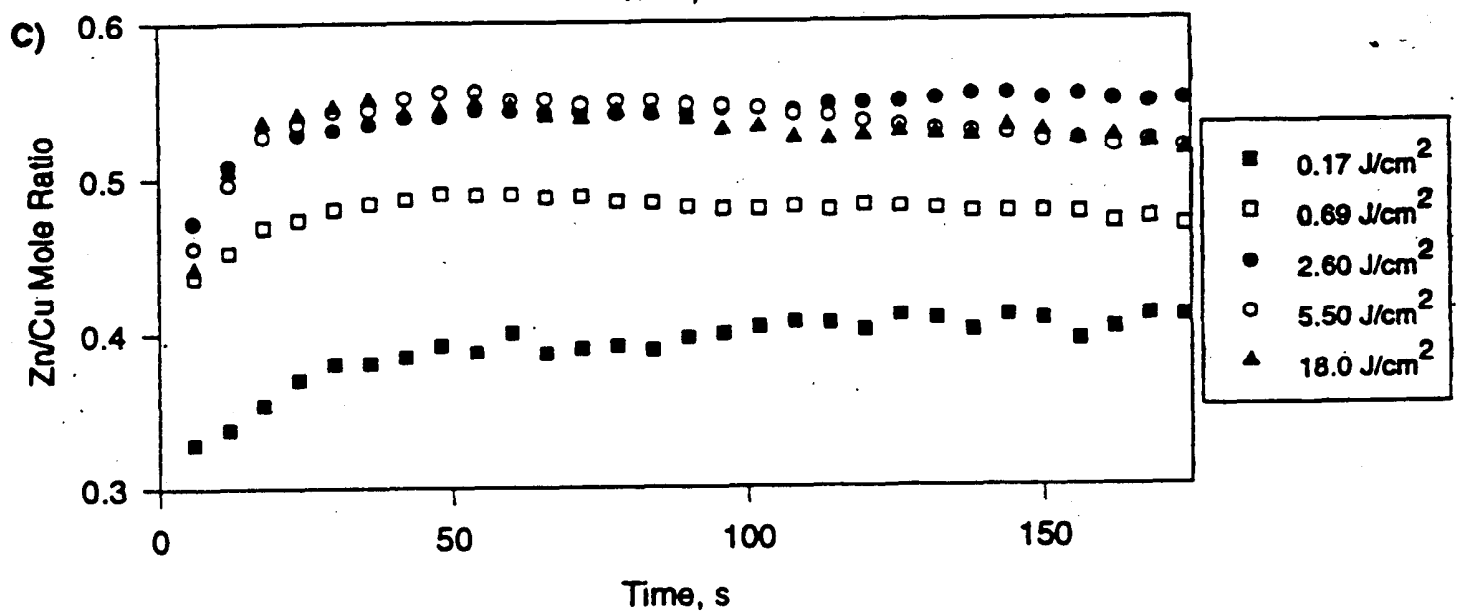

Figure $6 \mathrm{Zn}$-to- $\mathrm{Cu}$ mole ratios time profiles, measured during continuous ablation with 30-ns $\mathrm{KrF}$ excimer (A), 3-ns Nd:YAG (B), and 35-ps Nd:YAG (C) lasers at various fluences as indicated in the legends. 


\subsection{EFFECTS OF GAS ENVIRONMENT ON PICO-SECOND LASER ABLATION SAMPLING FOR ICP-AES}

During laser ablation, the sample is contained in a chamber with a flowing gas stream at atmospheric pressure. The laser beam passes through the gas atmosphere before reaching the sample surface. The gas atmosphere, therefore, may have a strong influence on the laser ablation process and affect the efficiency of laser sampling. In this part of the research, the effects of gas medium on picosecond laser ablation sampling were studied for enhancing ICP-AES sensitivity. Five noble gases were used in the laser ablation sampling chamber. These five gases represent a relatively large range of ionization potential (IP), atomic mass, and thermal conductivity. Since the temperature and excitation characteristics of a mixed gas ICP change with the gas type, analyte spectral line emission intensity and ICP vertical emission profile shape may also change. To ensure a valid comparison of the gas effects on laser ablation, ICP emission spectra and ICP vertical spatial profiles for laser sampling in different gases were measured and the effect relative to laser sampling in $\mathrm{Ar}$ was reported (Chan, under submission). Ar is used as the reference because it is the most commonly used carrier gas for the ICP and has a medium ionization potential among the five gases.

The experimental system consists of a picosecond Nd:YAG (1064 nm) for laser sampling, the inductively coupled plasma (ICP), and the imaging spectrometer with a charge coupled device (CCD) detector for ICP vertical profile emission intensity measurement. Laser power densities of 7 to $70 \mathrm{GW} / \mathrm{cm}^{2}$ were used. A stream of carrier gas passes through the chamber to deliver the laser-sampled material to the central channel of the ICP torch via a Teflon tube $(6 \mathrm{~mm}$ in diameter and $1 \mathrm{~m}$ in length) (cf. Figure 1). There are two carrier gas streams. One gas stream passes through the ablation chamber to sweep the laser-sampled material out of the chamber into the ICP. The second gas stream combines with the frost stream at the exit of the chamber via a Tjoint. The flow rates of both gases were $0.5 \mathrm{~L} / \mathrm{min}$, i.e., the combined gas flow rate was $1 \mathrm{~L} / \mathrm{min}$. The first stream of carrier gas that passes through the laser sampling chamber is called "chamber gas" ; the second stream is called " makeup gas."

Five noble gases ( $\mathrm{He}, \mathrm{Ne}, \mathrm{Ar}, \mathrm{Kr}$, and $\mathrm{Xe}$ ) were used. The noble gases were always paired with Ar as a makeup gas-chamber gas pair. Two identical sets of measurements for each gas pair were performed. The ICP-AES emission intensities were ratioed to determine the effect of the noble gas on laser sampling relative to Ar. Since the gas composition in the ICP was kept constant, the effects of gas medium on ICP excitation characteristics are canceled by ratioing the two intensities. All five possible pairs of gases were used, namely, $\mathrm{He}-\mathrm{Ar}, \mathrm{Ne}-\mathrm{Ar}, \mathrm{Ar}-\mathrm{Ar}, \mathrm{Kr}-\mathrm{Ar}$ and Xe-Ar.

Brass samples $(35 \% \mathrm{Zn}$ and $65 \% \mathrm{Cu})$ were used for the entire study. All samples were polished with 300-grit sand papers and cleaned with distilled-deionized water before sampling. For each measurement, a fresh spot on the sample surface was used. Each spot was pre-ablated for one minute (i.e. 600 laser pulses) to obtain a steady mass ablation rate before measurement of the ICP emission intensity.

Using $\mathrm{He}$ as the chamber gas, $\mathrm{Cu}$ I and $\mathrm{Cu}$ II are enhanced by 6 times, but $\mathrm{Zn}$ II is enhanced by 20 times. The difference in intensity ratios for $\mathrm{Cu}$ versus $\mathrm{Zn}$ is smaller using $\mathrm{Ne}, \mathrm{Ar}, \mathrm{Kr}$, and Xe: $10-20 \%$. There is 2 times enhancement in sensitivity in $\mathrm{Ne}$ and $20 \%$ reduction in sensitivity for Xe. These experiments demonstrate that the quantity of ablated mass can be significantly 
enhanced by using a small percentage of helium in the ablation chamber. An increased quantity of mass provides stronger analytical AES intensity, which in turn provides enhanced sensitivity.

\subsection{CONCLUSION}

In this initial year of research, we studied and demonstrated three very important components of laser ablation sampling for chemical characterization. In one study, we demonstrated that the ICP-AES technology is accurately responding to the chemical composition. The ratio of $\mathrm{Mg}$ ionic to atomic emission lines, and Fe atomic emission line pairs was used to confirm that ICP conditions do not change with different amounts of ablated mass. By changing the laser beam properties and thereby the ablation conditions, the amount of ablated mass was changed over approximately an order of magnitude. Under these experimental conditions, the ICP was not perturbed by different amounts of mass. The plasma conditions remained constant proving that the ICP can be used for accurate chemical analysis over a large range of laser operating conditions. Such a study has never been performed and clearly confirms the robustness of the ICP for representing laser ablated mass. With this information, analysts can rely on the concentration and composition changes measured in the ICP.

In a second study, we demonstrated how the quantity and composition of ablated mass changes during time as the laser repetitively removes mass from the sample. The quantity of ablated mass and its composition strongly depend on the number of laser pulses and laser fluence at the sample surface. It is important for the analyst to be aware of these effects as the reported chemical composition can be greatly influenced by time and laser power density.

Finally, we demonstrated how the efficiency of laser sampling can be improved by simply using a different gas in the ablation chamber. In this case helium provided the greatest enhancement. The noble gases ( $\mathrm{He}, \mathrm{Ne}, \mathrm{Ar}, \mathrm{Kr}, \mathrm{Xe}$ ) influence the ICP excitation characteristics and the sampling efficiency significantly. The significant enhancement in ICP sensitivity in $\mathrm{He}$ is due to a convolution of the effect of higher He mixed gas ICP temperature and laser sampling efficiency. The improvement in sampling efficiency is beneficial to chemical analysis especially for trace analysis which requires high sensitivity.

\subsection{MANUSCRIPTS RESULTING FROM THIS WORK AND SUBMITTED TO TECHNICAL $\begin{array}{lllllllll} & \mathbf{O} & \mathbf{O} & \mathbf{U} & \mathbf{R} & \mathbf{N} & \mathbf{A} & \mathbf{L} & \mathbf{S}\end{array}$}

A. C. Ciocan, X. L. Mao, Oleg V. Borisov and R. E. Russo. Optical Emission Spectroscopy Studies of the Influence of Laser Ablated Mass on Dry Inductively Coupled Plasma Conditions, submitted to Spectrochimica Acta, April 1997.

V. Borisov, X. L. Mao, A. C. Ciocan, and R. E. Russo. Time-Resolved Parametric Studies of Laser Ablation Using Inductively Coupled Plasma Atomic Emission Spectroscopy, submitted to Applied Surface Science, July 1997.

W.T. Chan, Amy P.K. Leung, X.L. Mao and R.E. Russo. Effects of gas environment on picosecond laser ablation sampling for ICP-AES, submitted to Applied Surface Science, July 1997. 

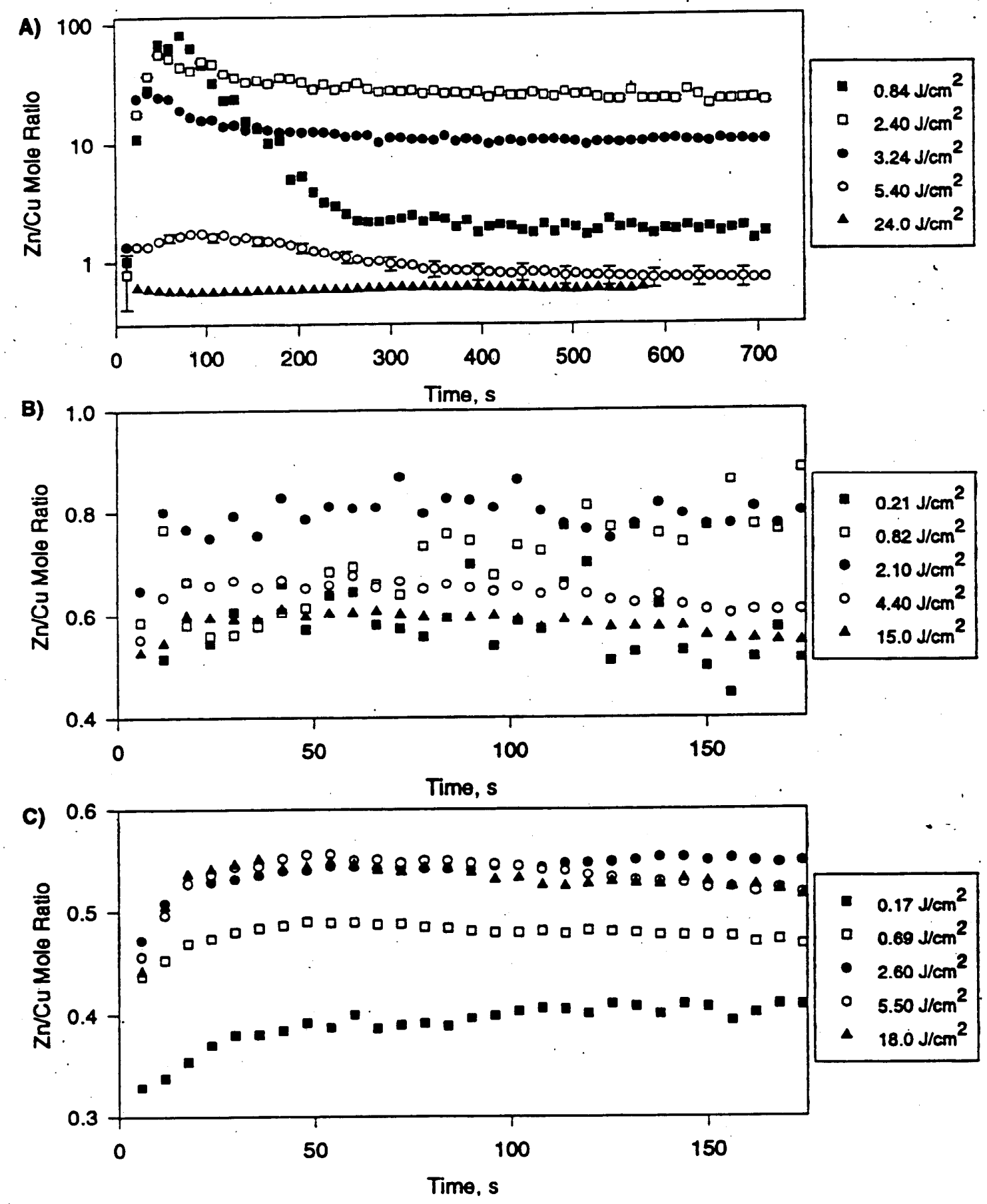

Figure 6. Zn-to-Cu mole ratios the profiles, measured during continuous ablation with 30-ns $\mathrm{KrF}$ excimer (A), 3-ns Nd:YAG (B), and 35-ps Nd:YAG (C) lasers at various fluences as indicated in the legends. 